\title{
Calculation of ionization in direct-frequency comb spectroscopy
}

\author{
B. Lomsadze, C. W. Fehrenbach, and B. D. DePaola* \\ J. R. Macdonald Laboratory, Department of Physics, Kansas State University, Manhattan, Kansas 66506-2601, USA
}

(Received 16 February 2012; published 6 April 2012)

\begin{abstract}
Direct-frequency comb spectroscopy (DFCS) is currently the highest-resolution absolute frequency spectroscopic technique known. In general, one does DFCS by scanning the repetition rate, $f_{\text {rep }}$, of a comb laser and measuring fluorescence from the excited states of the species under study. The technique has already been successfully characterized by a theoretical model that starts with the optical Bloch equations and, with a few simplifying assumptions converts them into linear coupled iterative equations. In the present work we build on that successful model to predict the characteristics of the ion yield from photoionization by the comb laser, as a function of $f_{\text {rep }}$. We show that the ion spectrum yields the same atomic structure as the fluorescence spectra, but with greater efficiency.
\end{abstract}

DOI: 10.1103/PhysRevA.85.043403

PACS number(s): 32.80.Rm, 82.53.Kp

\section{INTRODUCTION}

Direct-frequency comb spectroscopy (DFCS) is a powerful, relatively new technique for high-resolution spectroscopy on atomic or molecular systems [1-3]. In DFCS a mode-locked laser is run with its repetition rate locked to a precision rf frequency reference. The resulting broadband output has a spectrum composed of discrete narrow-band teeth. With the group velocity appropriately controlled to maintain a constant phase, $\phi$, the frequency of the $n$th tooth is given by

$$
f_{n}=n f_{\text {rep }}-f_{0} \text {, }
$$

where $f_{\text {rep }}$ is the repetition rate of the laser, $f_{0}=f_{\text {rep }}(2 \pi \phi)^{-1}$ is the offset frequency, and $n$ is an integer. The teeth frequencies are stable and constant in time. As explained in several review articles [3,4], DFCS measurements are made by scanning either $f_{\text {rep }}$, or $f_{0}$, and observing fluorescence whenever a comb tooth is resonant with a transition in the target system. Because the frequency of the comb tooth is known $\left(\bmod f_{\text {rep }}\right)$ from Eq. (1), one can, in principle, deduce the absolute frequency of that transition with extremely high precision.

Some complications arise because the fluorescence is often at the same frequency as the comb laser and care must therefore be taken to avoid background from scattered laser light. Nevertheless, excellent quality data have been taken in this manner [2,5].

Perhaps even more interesting, two-photon excitation can occur when the frequencies of two comb teeth sum to the frequency difference between states via a nonresonant transition to an intermediate state. At first, this type of transition seems unlikely, due to the nonresonant character of the intermediate transition. However, as Ye's group has pointed out [1], if one pair of teeth is two-photon resonant, then thousands of other pairs of teeth will also be resonant with that same transition. For example, if $f_{n}$ and $f_{m}$, combine to be two-photon resonant then so will $f_{n+1}+f_{m-1}$ and $f_{n+2}+f_{m-2}$, etc.

In a prototypical system, ${ }^{87} \mathrm{Rb}$, the relevant manifolds of states, enumerated as 1 through 3 , are the $5 s, 5 p$, and $5 d$,

*Corresponding author: depaola@phys.ksu.edu respectively. When a comb tooth is shifted into resonance with a transition between states in the $5 s$ and $5 p$ manifolds, for example from $5 s_{1 / 2}, F=2$ to $5 p_{3 / 2}, F=3$, then the upper state $\left(5 p_{3 / 2}, F=3\right)$ will be populated, and a fluorescence signal from the $5 p$ manifold can be detected. In this example, both excitation and fluorescence wavelengths are near $780 \mathrm{~nm}$, necessitating severe spatial filtering to avoid excessive background signal on the detector. However, for the two-photon excitation process, a convenient feature of the ${ }^{87} \mathrm{Rb}$ system is that the levels in the $5 d$ manifold can cascade decay through the $6 p$ manifold, and from there to the ground state, emitting light at $420 \mathrm{~nm}$. Ye's group measured the $420 \mathrm{~nm}$ light as a function of either $f_{0}$ or $f_{\text {rep }}$, obtaining absolute energies, with extremely high precision, of all the states in the $5 d$ manifold.

A possible alternative to measuring fluorescence in DFCS experiments is to detect ions. Detecting ions has some advantages. First, ion detection is far more efficient than photon detection: Use of weak electric fields gives $4 \pi$ sr ion collection and ion detectors are typically more efficient than photon detectors. (If Stark effects from even weak fields are a problem, the extraction electric field can be pulsed.) Furthermore, although in the original experiment [1] the photons detected after two-photon excitation were at a different wavelength from the laser, this will not be the case in general. For example if one wants to study the structure of the $\mathrm{Rb} 4 d$ states using two different combs, one comb (centered at $780 \mathrm{~nm}$ ) used for excitation from $5 s$ to $5 p$, and the second (centered at $1529 \mathrm{~nm}$ ) from $5 p$ to $4 d$, the photons resulting from decay will have the same wavelength as the lasers because the decay and excitation pathways are identical. In contrast background counts should be negligible in appropriately designed ion detectors.

One method for producing ions is to use a second laser to photoionize the comb-excited states [6]. However, ions can be produced by photoionization by the same comb laser that produced the excitation. This is the phenomenon investigated here. We simulate a DFCS experiment in which we include ionization by the comb laser. We show calculated $5 p, 5 d$, and ionization continuum populations as functions of $f_{\text {rep }}$ for a fixed value of $f_{0}$. In Sec. II the model is described. In Sec. III the results of the model are presented and discussed. In Sec. IV we conclude with a summary of the ionization technique, and propose a specific experiment to test the model. 


\section{MODEL DESCRIPTION}

The calculation is based on the model by Felinto [7,8]. The Felinto model starts with the optical Bloch equations for excitation of rubidium $5 s, 5 p$, and $5 d$ manifolds. By manifold is meant the full structure with fine and hyperfine levels; those three manifolds include a total of 16 states when summed over $m_{F}$. The system is then subjected to a train of equally (temporally) spaced optical pulses, all identical, except for a fixed pulse-to-pulse phase shift, $\phi$. The Bloch equations are not integrated over the entire interaction time. Rather, the excitation is calculated for a single laser pulse and the effects of multiple pulses are added coherently using an iterative algorithm with incoherent redistribution of population by spontaneous decay occurring between pulses. It is assumed that the incoherent redistribution of populations (spontaneous emission) occurs on a timescale that is long compared to that of coherent excitation (the temporal length of the optical pulse). It is also assumed the time between pulses is long compared to the width of the pulses.

In the formulation developed by Felinto, one can compute the time evolution of the populations of each state in all three manifolds to any chosen level of perturbation, at a computation cost that is linear in degree of perturbation. In Felinto's (and our) computer code, computer roundoff limits us to twelfthorder perturbation [9], which already is at the low end of the strong field regime.

For this work we want to include photoionization of the $5 d$ states, so two extra manifolds were added to the model, the continuum, labeled 4 , and holding states, labeled 5 . The continuum is modeled by 26 discrete energy levels. Each level is composed of 14 energy-degenerate angular momentum states, one for every possible angular momentum required to allow a transition from each of the 8 states in the $5 d$ manifold. The 26 discrete levels are spaced $2 \mathrm{THz}$ apart in energy, for a total frequency spread in the manifold of $50 \mathrm{THz}$ (or about $100 \mathrm{~nm}$ at the laser's central wavelength), allowing us to span the bandwidth of the excitation laser. Manifold 4 lies above manifold 3 by an energy equivalent to the central frequency of the comb laser. All the angular portions of the dipole matrix elements for excitation to the continuum manifold were computed using the usual angular momentum algebra, thereby guaranteeing orthonormality. The reduced matrix element for ionization was estimated using the $\operatorname{Rb}(5 d)$ photoionization cross section measured [10] using a cw laser with frequency near that of the center of the comb envelope. The photoionization cross section is expected [10] to vary little over the bandwidth of the comb envelope frequency.

Simply modeling a continuum in this fashion is unsatisfactory because the levels are still discrete and the model would therefore not predict ionization if the photon energies added to something between the discrete levels. Furthermore, states in the continuum manifold must be treated differently from bound states: ions cannot spontaneously decay back down to bound states. And while it is possible for an atom excited to the ion continuum to undergo stimulated emission in the same short laser pulse, it is unrealistic to allow electrons ionized with one pulse to be stimulated back down to the bound state with the next pulse, several nanoseconds later.

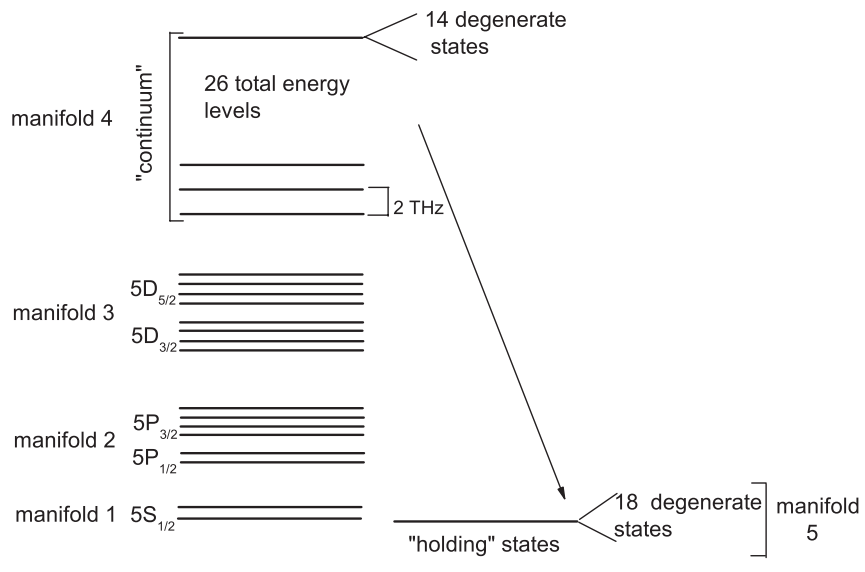

FIG. 1. Simplified energy level diagram for ${ }^{87} \mathrm{Rb}$. Individual states are grouped into manifolds. The continuum is modeled by manifolds 4 and 5 . See text for details.

In order to address these two issues and make our code more realistic we added a fifth manifold to our model, a so-called holding manifold. This manifold consists of 18 degenerate states, one for every possible angular momentum required to allow a transition from each of the continuum states. The single energy of these 18 states was chosen to lie a couple of laser bandwidths below the continuum states and are coupled to the continuum states only through spontaneous emission. The decay rate for the continuum states, $\Gamma$, was chosen to be $2 \mathrm{THz}$. That is, the line width of the continuum levels is equal to the spacing between adjacent levels. Furthermore, atoms that are excited to the continuum decay to the holding manifold on a timescale consistent with a true ionization process. In total, then, 398 discrete states are used to model the $\mathrm{Rb}$ atom and its continuum: 2 in manifold 1, 6 in manifold 2, 8 in manifold 3, $14 \times 26=350$ in manifold 4, and 18 in manifold 5. A partial energy level diagram of the model ${ }^{87} \mathrm{Rb}$ system is shown in Fig. 1.

In the original Felinto model, one of the approximations made was that incoherent redistribution occurs on a timescale that is long compared with that of coherent excitation [8]. Therefore in that work, terms containing $\Gamma_{i j}$, the decay rate between levels $i$ and $j$, could be removed from their integrals and neglected during the pulse. It is clear that in our model for which the $\Gamma$ 's of the continuum states equal $2 \mathrm{THz}$, this approximation is no longer valid. Therefore we numerically integrate these terms. The only additional approximation we are making in this model is to ignore excitation and ionization from the $7 s$ states, which could be populated with one photon excitation from the $5 p$ states. Justification for this approximation is that the $5 p \rightarrow 7 s$ transition wavelengths are on the edge of the laser bandwidth of a typical Ti:sapphire comb laser and correspondingly their contribution to the ionization signal should be negligible. Nevertheless, at some later time it may prove interesting to include the $7 \mathrm{~s}$ states in the calculation to see what role they may play in ion production.

The initial repetition rate of the comb laser in the computation was $75557551 \mathrm{~Hz}$, which is typical of the comb used in our laboratory. We define this frequency to be $f_{\text {ref }}$. Most calculations were made using 950 pulses in the train. This 
seems to be a good compromise [8] between the narrowing of the comb teeth that more pulses cause, and broadening of the resonances from the incoherent process of spontaneous emission.

\section{RESULTS}

Figure 2 shows the result of a typical calculation. Here, relative populations in the $5 p, 5 d$, and $\mathrm{Rb}^{+}$(continuum plus holding) manifolds are plotted versus $f_{\text {rep }}-f_{\text {ref }}$. Though their heights vary over several orders of magnitude, each peak in the $5 d$ and ion curves has been identified as resulting from a specific two-photon transition from the $5 s \rightarrow 5 d$ manifolds; a few representative peaks are labeled in the figure. The individual laser pulses were hyperbolic secant, having temporal widths of $50 \mathrm{fs}$, and peak intensities of $10^{4} \mathrm{~W} \mathrm{~cm}^{-2}$. The central wavelength of the laser was $778.6 \mathrm{~nm}$, and the offset frequency was set to $f_{0}=14.5 \mathrm{MHz}$. Several aspects of Fig. 2 are noteworthy. First, every line in the $5 d$ spectrum has a corresponding line in the ion spectrum, and vice versa. That is, the ion signal has exactly the same information content as the photon signal. It should also be noted that the ion population is nearly an order of magnitude greater than the $5 d$ population. This does not indicate that ionization is significantly depleting the atomic population. Rather, what it shows is that once an ion is formed it cannot relax back into a neutral state. Therefore, ions accumulate throughout the 950 pulse interrogation time. The $5 d$ states, on the other hand, are constantly decaying and being re-excited. An estimate of the number of photons that are

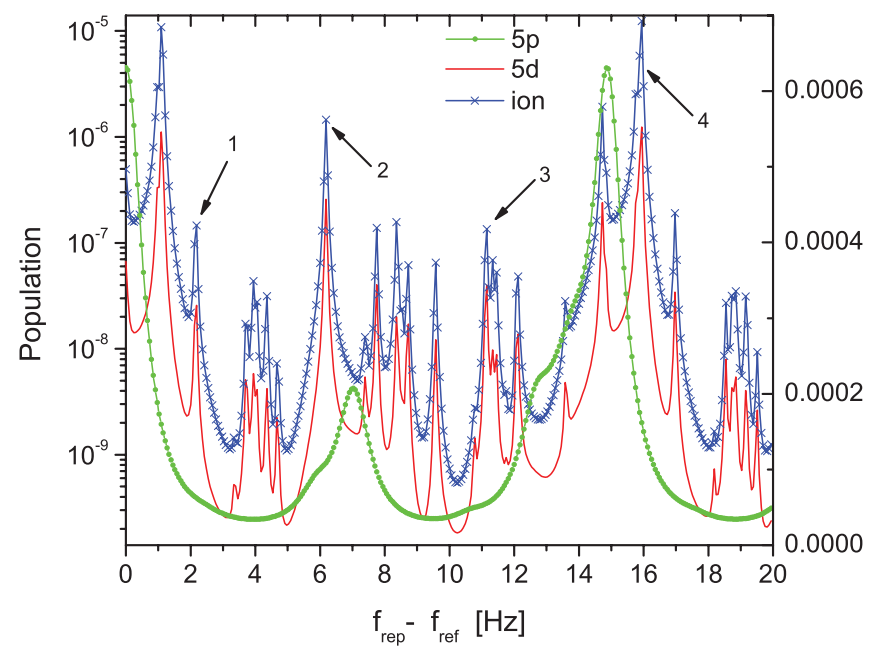

FIG. 2. (Color online) Plot of the relative populations in the $5 p$, $5 d$, and $\mathrm{Rb}^{+}$manifolds as functions of the comb laser repetition frequency, minus a fixed reference frequency, $f_{\text {ref }}=75557551 \mathrm{~Hz}$. The $5 p$ population is plotted as round points connected by a line (green online) and uses the linear scale on the far right; the $5 d$ is plotted as a solid line without points (red online) and uses the log scale on the left; and the $\mathrm{Rb}^{+}$population is plotted as crosses connected by a line (blue online) and also uses the log scale on the left. All of the peaks in the $5 d$ and ion curves have been identified as resulting from two-photon transitions from the $5 s \rightarrow 5 d$ manifolds. A few selected peaks are labeled as (1) $5 s_{1 / 2}, F=1 \rightarrow 5 d_{5 / 2}, F=2$; (2) $5 s_{1 / 2}, F=$ $2 \rightarrow 5 d_{5 / 2}, F=2$; (3) $5 s_{1 / 2}, F=1 \rightarrow 5 d_{5 / 2}, F=1$; (4) $5 s_{1 / 2}, F=$ $2 \rightarrow 5 d_{5 / 2}, F=4$. produced on the $6 p \rightarrow 5 s$ transition from cascade decays of the $5 d$ states shows that an atom would emit, on average, about five photons at $420 \mathrm{~nm}$ during this interrogation time. Thus, the photon yield and ion yield are quite comparable for these typical laser intensities. However, the detection efficiencies for ions and photons can differ substantially.

One might expect additional structure in the ion spectra from two-photon ionization of atoms in the $5 p$ states. That is, once an atom has been excited to a $5 p$ state, virtually any pair of comb teeth is resonant with two-photon ionization to a continuum. This two-photon process is similar to the one that excites states from the $5 s$ to the $5 d$ manifold, but should occur when a comb tooth has a frequency resonant with a $5 s \rightarrow 5 p$ transition. However, the ion signal shows no structure corresponding to direct $5 p$ excitation, followed by two-photon ionization.

To understand the reason for the lack of $5 p$ structure in the ionization spectrum we tried to estimate the probability for direct two-photon ionization of the $5 p$ states. To do this we modified our code, prohibiting spontaneous emission from the $5 p$ states. (Stimulated emission and absorption were still permitted.) We then set the initial population of the $5 p$ manifold to vary sinusoidally with $f_{\text {rep }}$, with its population shared equally between all states in the manifold. The remainder of the population was equally split between the two states in the $5 s$ manifold. The result of this calculation is shown in Fig. 3, where we plot the ion population as a function of $f_{\text {rep }}$. Clearly, the ion population follows the $5 d$ population, even though the $5 p$ population is many orders of magnitude larger than that of the $5 d$ manifold. However, we can also see that the ion population does not have the detailed structure contained in the $5 d$ spectrum, but rather has the smooth profiles of the $5 p$ spectrum. Based on this calculation we estimate that,

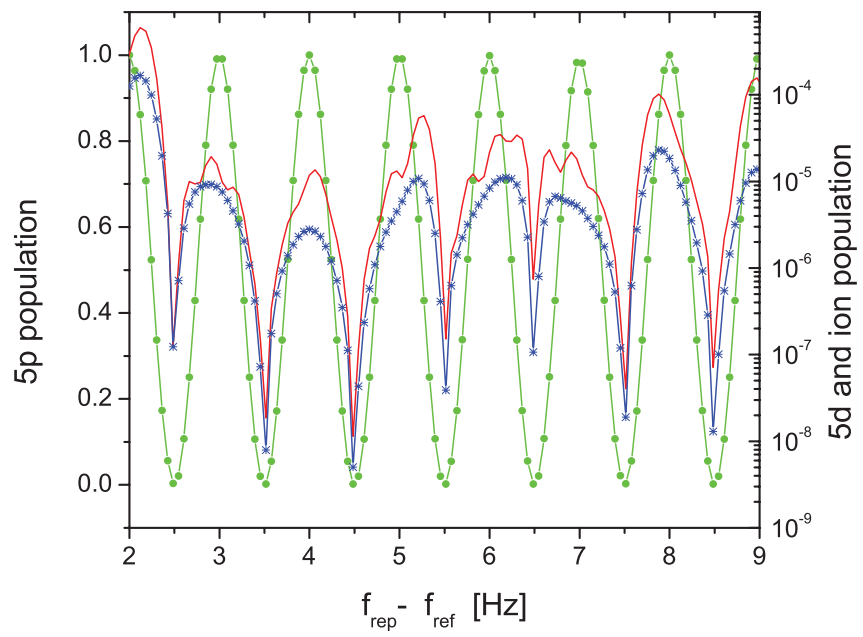

FIG. 3. (Color online) Computation of $5 d$ (solid line without points, red online) and ionization (solid line with crosses, blue online) manifolds under the artificial initial condition that the initial population of $5 p$ states (solid line with circular points, green online) varies sinusoidally with $f_{\text {rep }}$. The ionization population largely follows the $5 d$ population. However, the lack of $5 d$ structure in the ionization population is an indication of two-photon ionization of states in the $5 p$ manifold (without being resonant with an intermediate $5 d$ state). 


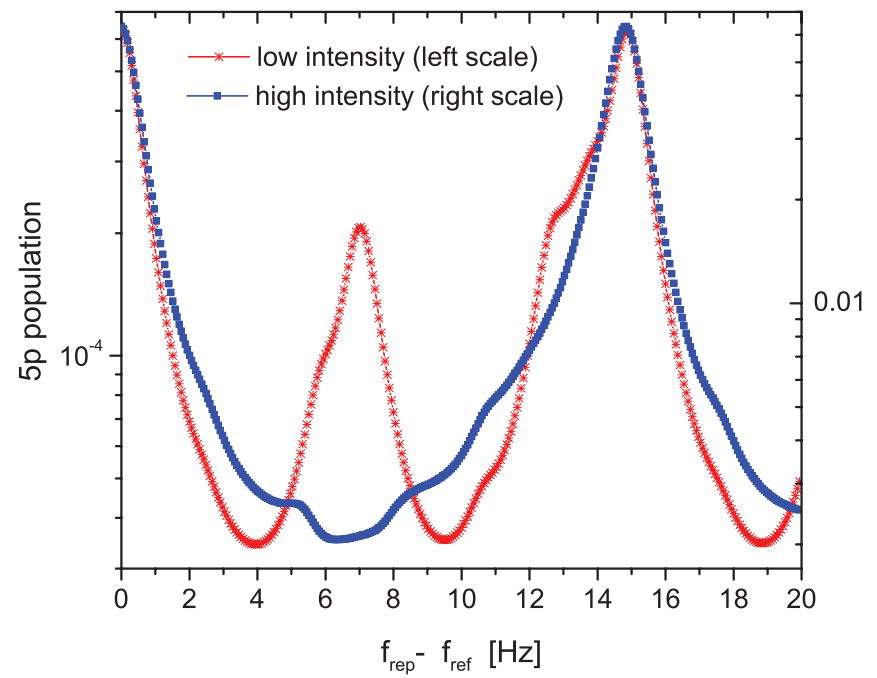

FIG. 4. (Color online) Detail of $5 p$ population for low- and highintensity comb laser. The peak near $7 \mathrm{~Hz}$ is from the $5 s_{1 / 2}, F=$ $2 \rightarrow 5 p_{3 / 2}, F=2$ transition and clearly shows the effects of optical pumping. The peak near $15 \mathrm{~Hz}$ is from the $5 s_{1 / 2}, F=2 \rightarrow 5 p_{3 / 2}, F=$ 3 transition, for which no optical pumping is expected.

for the intensity used in these computations, the probability of two-photon ionization from the $5 p$ states is on the order of $10^{-5}$. Recognizing that this analysis is crude, we nevertheless can say that for the realistic calculations typified by Fig. 2, when a comb tooth is resonant with a $5 s \rightarrow 5 p$ transition, the population of the $5 p$ manifold is about $10^{-4}$, and that the resulting two-photon ionization should be on the scale of $10^{-9}$, consistent with us not seeing it in the spectra.

A related question is why is the $5 p$ population so small? The absence of nearly all direct $5 p$ excitation from $5 s$ is readily explained by optical pumping. In Fig. 4 we plot the population of the $5 p$ manifold as a function of $f_{\text {rep }}-f_{\text {ref }}$ for two different laser intensities. We see that the peak corresponding to the $5 s_{1 / 2}, F=2 \rightarrow 5 p_{3 / 2}, F=2$ transition disappears when the intensity of the laser is increased by a factor of 10 . Further evidence of optical pumping is shown in Fig. 5(a) where we plot the $5 s$ hyperfine levels $F=1$ and $F=2$ as a function of $f_{\text {rep }}-f_{\text {ref }}$. For the lower intensities, optical pumping is not noticeable but by increasing the intensity by a factor of 10 almost all the population is pumped from $F=1$ to $F=2$. Therefore, attempting to see the $5 p$ population by increasing the laser intensity, results in increased optical pumping to an inaccessible $5 s$ hyperfine level. The only two transitions in this system for which optical pumping does not take place are $5 s_{1 / 2}, F=2 \rightarrow$ $5 p_{3 / 2}, F=3$ and $5 s_{1 / 2}, F=1 \rightarrow 5 p_{3 / 2}, F=0$.

As Eq. (1) shows, the frequency of the $n$th comb tooth depends on both $f_{\text {rep }}$ and $f_{0}$. This means that it should be possible to have one comb tooth resonant with, say, the $5 s_{1 / 2}, F=2 \rightarrow 5 p_{3 / 2}, F=2$ transition, and a second tooth resonant with the $5 s_{1 / 2}, F=1 \rightarrow 5 p_{3 / 2}, F=2$ transition. In this case, no optical pumping should take place [11]. In Fig. 5(c) (low intensity) and Fig. 5(d) (high intensity) we plot the $5 s_{1 / 2}$ hyperfine levels $F=1$ and $F=2$ as a function of $f_{\text {rep }}-f_{\text {ref }}$, but now with a value of $f_{0}$ chosen to negate optical pumping. The contrast between Figs. 5(b) and 5(d) is obvious.

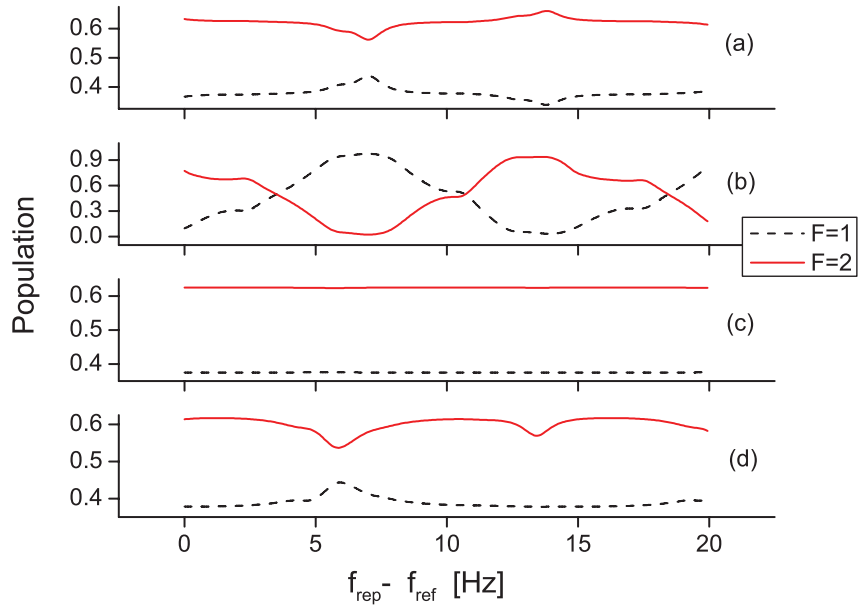

FIG. 5. (Color online) Ground-state hyperfine populations versus $f_{\text {rep }}$ when a comb tooth is resonant with the $5 s_{1 / 2}, F=2 \rightarrow$ $5 p_{3 / 2}, F=2$ transition. (a) is for low comb laser intensity and does not exhibit strong optical pumping. In (b) the comb laser intensity is high and strong optical pumping from $5 s_{1 / 2}, F=2$ to $5 s_{1 / 2}, F=1$ is clearly seen. (c) and (d) are the same intensity conditions as (a) and (b), respectively, but $f_{0}$ has been adjusted such that a second comb tooth is resonant with the $5 s_{1 / 2}, F=2 \rightarrow 5 p_{3 / 2}, F=2$ transition. The optical pumping is minimal, as expected.

We next varied the number of pulses in the train. Computation time is linear in the number of pulses in the train; if we can decrease the number of pulses in the train without significantly affecting the spectra, then the computations can be done more readily. However, as the number of pulses decreases the width of the comb teeth grows, leading to a broadening of the structure in our spectra [8]. Furthermore, as already demonstrated [8], less pulses in the train means less overall population movement. Figure 6 shows the same calculation as in Fig. 2 but with 50 pulses in the train. One can see that the

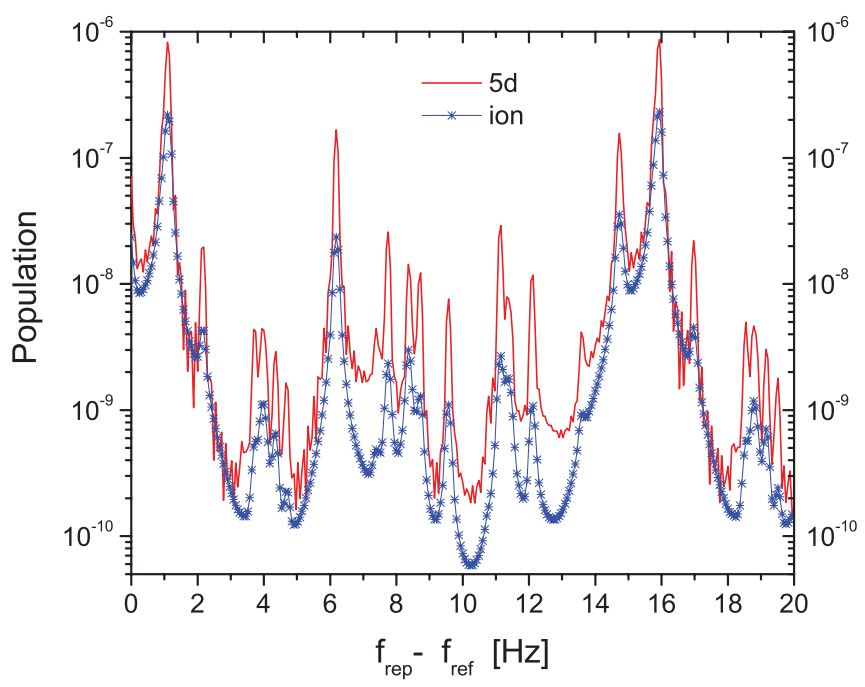

FIG. 6. (Color online) The same as Fig. 2, but for 50 pulses in the train. The peaks are slightly broader and ringing is seen in the populations. The ringing is actually due to the Fourier transform of a rectangular pulse, superimposing a sinc function on the comb teeth, and is especially evident on the $5 d$ curve. 
structure is broader and the excitation or ionization decreased, as expected. However Fig. 6 still reflects the essential features of the $\mathrm{Rb}$ atom's structure, but with about $5 \%$ of the computer time (a couple of hours on our system). In passing, we note that the $5 d$ spectrum, for example, has additional ringing on it compared to the corresponding spectrum in Fig. 2. The ringing exists because the Fourier transform of a finite pulse train is the convolution of the Fourier transform of an infinite train (an ideal comb) with the Fourier transform of a square pulse having temporal width equal to the number of pulses in the

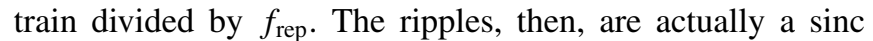
function on the comb teeth and are reflected in the excitation spectra.

\section{CONCLUSION}

In this work we expanded the Felinto formalism to include photoionization in an atomic system interacting with an optical frequency comb. We made calculations for $\mathrm{Rb}$ that showed once the $5 d$ states are populated by two-photon excitation, they are efficiently ionized by the comb. For the laser parameters we used, which are typical of Ti:sapphire combs, we found the ion yield from exciting a $5 d$ state to be comparable to the net photon yield from the $6 p \rightarrow 5 s$ cascade decay that would be produced by the same comb excitation. Since we can collect the ions over $4 \pi$ sr with nearly unit efficiency and no background, collecting ions will be a significant improvement in the DFCS technique. Our calculations also showed that ion production tracks excitation of the $5 d$ state. Thus, detection of ions gives the same spectroscopic information as detection of fluorescence. In particular, we showed that the nonresonant, two-photon photoionization of the intermediate $5 p$ state is not a significant contribution to the ion signal and produces no new features. Another thing our calculations showed, which should have occurred in previous DFCS measurements in Rb but which was not reported, is that there should be strong optical pumping on the transitions out of the split ground state. This optical pumping inhibits one-photon excitation of the lowest excited state and can impact interpretation of DFCS results. By careful choice of $f_{0}$ and $f_{\text {rep }}$, it is possible to have a second comb tooth negate the optical pumping.

While the calculations were done on the atomic ${ }^{87} \mathrm{Rb}$ system, the results are completely general and indicate that measurement of the ionization signal can be advantageous for a great many systems of interest. The theory could be further enhanced by adding a cw laser to the system to study the combined $\mathrm{cw}$ and pulsed laser interaction with matter [12].

\section{ACKNOWLEDGMENTS}

The authors would like to express their deepest appreciation to Professor D. Felinto for the generous donation of his time and his computer code that served as the starting point from which our code evolved. We are also profoundly grateful to Professor O. L. Weaver for many extremely helpful discussions.
[1] A. Marian, M. C. Stowe, J. R. Lawall, D. Felinto, and J. Ye, Science 306, 2063 (2004).

[2] A. Marian, M. C. Stowe, D. Felinto, and J. Ye, Phys. Rev. Lett. 95, 023001 (2005).

[3] M. C. Stowe, M. J. Thorpe, A. Pe'er, J. Ye, J. E. Stalnaker, V. Gerginov, and S. A. Diddams, Adv. At. Mol. Opt. Phys. 55, 1 (2008).

[4] J. Ye and S. T. Cundiff, Femtosecond Optical Frequency Comb: Principle, Operation, and Applications ( Springer, Berlin, 2005).

[5] V. Gerginov, C. E. Tanner, S. A. Diddams, A. Bartels, and L. Hollberg, Opt. Lett. 30, 1734 (2005).

[6] S. Witte, R. T. Zinkstok, W. Ubachs, W. Hogervost, and K. S. E. Eikema, Science 307, 400 (2005).
[7] D. Felinto, L. H. Acioli, and S. S. Vianna, Phys. Rev. A 70, 043403 (2004).

[8] D. Felinto and C. E. E. López, Phys. Rev. A 80, 013419 (2009).

[9] This limitation can be somewhat relaxed through a different choice of precision in the numbers stored to disk between separate parts of the computation.

[10] B. C. Duncan, V. Sanchez-Villicana, P. L. Gould, and H. R. Sadeghpour, Phys. Rev. A 63, 043411 (2001).

[11] We do not consider optical pumping between $m_{F}$ states, for which polarization control is also needed.

[12] J. E. Stalnaker, Y. LeCoq, T. M. Fortier, S. A. Diddams, C. W. Oates, and L. Hollberg, Phys. Rev. A 75, 040502 (2007). 